\title{
Odontogenic tumors: a review of 60 cases
}

\author{
Ajinkya Varkhede ${ }^{1}, J V$ Tupkari ${ }^{2}$, MS Mandale ${ }^{3}$, Manisha Sardar ${ }^{4}$ \\ ${ }^{1}$ P.G. Student, Government Dental College \& Hospital, Mumbai, Maharashtra, India. \\ ${ }^{2}$ Prof. and H.O.D., Government Dental College \& Hospital, Mumbai, Maharashtra, India. \\ ${ }^{3}$ Associate Professor, Government Dental College \& Hospital, Mumbai, Maharashtra, India. \\ ${ }^{4}$ Senior Lecturer, Government Dental College \& Hospital, Mumbai, Maharashtra, India.
}

Correspondence:

Department of Oral Pathology and Microbiology,

Room no 301, Government Dental College and Hospital,

Mumbai 400001 Maharashtra,

India

Email address-varkhedeajinkya260@gmail.com

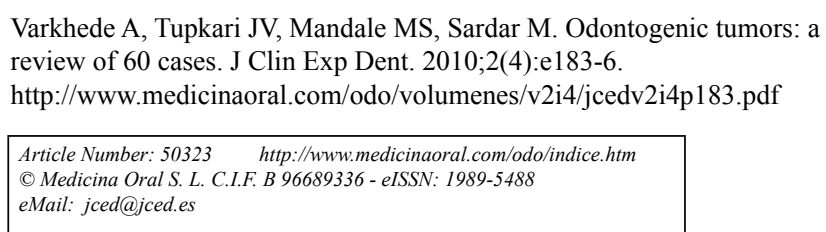

\begin{abstract}
Objective: Studies on odontogenic tumors published from many parts of the world show a distinct geographic variation; however, there is little information available in the English-language literature on the relative frequency of odontogenic tumors in India. This retrospective study was designed to determine the relative frequency of odontogenic tumors in an Indian population and compare them with various reports from other parts of the world. Study design: The histopathology records of the Department of Oral pathology and Microbiology of Government Dental College and Hospital, Mumbai were retrieved retrospectively within the period of January 2001 to March 2010. A total of 60 lesions classified as odontogenic tumors were reviewed. These were analyzed for age, gender, site of tumor and histopathologic typing. Criteria used were World Health Organization (WHO) classification 2005. The controversy still exists regarding the nature of odontogenic keratocyst or tumor, so it has not been included in this study.

Results: A total of 60 cases of odontogenic tumors were reported in this period. The most frequent histological type was ameloblastoma (66.67\%), followed by odontome (20\%), adenomatoid odontogenic tumor (10\%). In general, the odontogenic tumors showed a predilection for the mandible and the posterior regions of the jaws. Ameloblastomas occurred with a marked predilection for the mandible, while adenomatoid odontogenic tumor showed predilection for the maxilla, anterior regions of the jaws, and young females.

Conclusion: Odontogenic tumors show a definite geographic variation. In our study, ameloblastomas were the most frequent odontogenic tumors, with distinct anatomic predilections.
\end{abstract}

Key words: Odontogenic tumors, epidemiology, geographic distribution, prevalence, ameloblastoma. 


\section{Introduction}

Odontogenic tumors (OT) are a group of heterogeneous lesions derived from epithelial or ectomesenchymal tissues or both, which are part of the tooth-forming apparatus. They range from hamartomatous or nonneoplastic tissue proliferations to malignant neoplasms with metastatic capacity. In humans, tumors of the odontogenic tissues are comparatively rare, comprising about $1 \%$ of all oral and maxillofacial biopsy specimens diagnosed (1). Several retrospective studies carried out in Africa, Asia, Europe, and America, show that differences exist in the relative frequency of the various histologic types. (1-13)

Available literature on the relative frequency of odontogenic tumors are mostly among Americans and Africans. Very few studies are reported among Asians, especially from the Indian subcontinent. The aim of the present study was to determine the epidemiology and clinicopathologic presentation of this heterogeneous group of lesions seen at the Government Dental College and Hospital, Mumbai, India, over the period of January 2001 to March 2010 and to compare these data with previous reports.

\section{Materials and Methods}

The pathology records of the Department of Oral Pathology and Microbiology of Government Dental College and Hospital, Mumbai, India, were reviewed retrospectively for all of the lesions of the oral cavity and jaws seen from January 2001 to March 2010. A total of 60 lesions were classified as intraosseous odontogenic tumors during this period. All cases were analyzed for age, gender, site of tumor, and histopathologic typing.

The maxillary lesions were divided into 2 categories based on the radiographic extent. Class 1 consisted of lesions limited to the anterior segment of maxilla (distal aspect of right canine to distal aspect of left canine). Class 2 consisted of lesions limited to the posterior segment of maxilla (from mesial aspect of first premolar distally).

Similarly, the mandibular lesions were divided into 2 categories. Class 1 consisted of lesions limited to the ante-

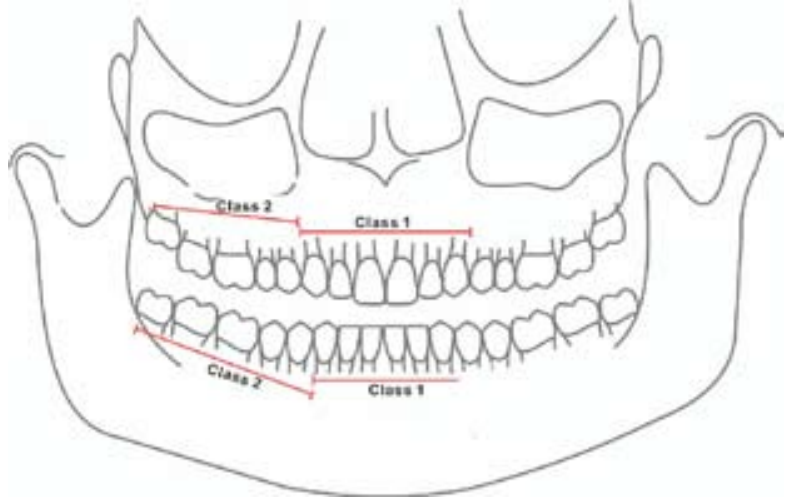

Fig.1. Anatomic division of maxilla and mandible into 4 regions. rior segment of mandible (distal aspect of right canine to distal aspect of left canine). Class 2 consisted of lesions limited to the posterior segment of mandible (mesial aspect of first premolar to distal aspect of third molar and beyond) Fig.1.

\section{Results}

A total of 60 cases with odontogenic tumors were seen and diagnosed during the period from January 2001 to March 2010. Of the 60 odontogenic tumors, all were benign. Among these, 30 were in males and 30 in females, male: female ratio of $1: 1$.

43 tumors were encountered in the mandible and 17 in the maxilla, with an overall mandible: maxilla ratio of 2.5:1.

Table 1 shows the frequency, gender, and site distribution for different pathologic types of tumors listed according to the WHO International Classification of Odontogenic Tumors.

\begin{tabular}{|l|c|c|c|c|c|}
\hline & \multicolumn{2}{|c|}{ Total } & \multicolumn{3}{c|}{ Gender } \\
\hline & Number & Percentage & Male & Female & Male:Female \\
\hline Ameloblastoma & 40 & $66.67 \%$ & 24 & 16 & 1.5 \\
\hline CEOT & 1 & $1.67 \%$ & 0 & 1 & NA \\
\hline AOT & 6 & $10 \%$ & 3 & 3 & 1 \\
\hline Ameloblastic fibroma & 1 & $1.67 \%$ & 0 & 1 & NA \\
\hline odontoma & 12 & $20 \%$ & 3 & 9 & 0.33 \\
\hline Total & 60 & & 30 & 30 & 1.1 \\
\hline
\end{tabular}

CEOT: Calcifying epithelial odontogenic tumor.

AOT: Adenomatoid odontogenic tumor.

NA: Not appliacble

Table 1. Frequency, gender, and site distribution of odontogenic tumors

Ameloblastomas were the most common benign tumors (66.67\%), followed by odontome (20\%), adenomatoid odontogenic tumor (10\%). Gender analysis showed a female predilection for most of the tumors except ameloblastoma.

Table 2 shows the age distribution of all of the odontogenic tumors found in this study.

Age distribution showed a peak occurrence in the third decade, $83.33 \%$ of the cases occurring between the second and fifth decades. Ameloblastoma, the most common tumor in this study showed $77 \%$ of the cases occurring in the third to fifth decades. Odontomes and adenomatoid odontogenic tumors (AOT) showed a peak occurrence in the second decade, with $100 \%$ and $75 \%$, respectively, of the tumors occurring in the second to third decades.

Table 3 shows the distribution of odontogenic tumors by site of occurrence.

The mandible was clearly the more common site of occurrence for most odontogenic tumors, with a ratio of 2.3:1. Ameloblastoma, showed a very high predilection for the mandible with $85 \%$ of the cases occurring in the mandible with a ratio of 5.67:1. It is worth mentioning that cases of adenomatoid odontogenic tumor had a predilection for the maxilla. In general, the odontogenic tu- 


\begin{tabular}{|l|c|c|c|c|c|c|c|c|}
\hline Tumor/ Age & $\mathbf{0 - 9}$ & $\mathbf{1 0 - 1 9}$ & $\mathbf{2 0 - 2 9}$ & $\mathbf{3 0 - 3 9}$ & $\mathbf{4 0 - 4 9}$ & $\mathbf{5 0 - 5 9}$ & $\mathbf{6 0 - 6 9}$ & Total \\
\hline Ameloblastoma & 0 & 4 & 12 & 12 & 7 & 3 & 2 & 40 \\
\hline CEOT & 0 & 0 & 0 & 1 & 0 & 0 & 0 & 1 \\
\hline AOT & 0 & 5 & 1 & 0 & 0 & 0 & 0 & 6 \\
\hline Ameloblastic fibroma & 0 & 0 & 1 & 0 & 0 & 0 & 0 & 1 \\
\hline odontoma & 1 & 5 & 4 & 0 & 1 & 0 & 1 & 12 \\
\hline
\end{tabular}

CEOT: Calcifying epithelial odontogenic tumor.

AOT: Adenomatoid odontogenic tumor.

Table 2. Distribution of odontogenic tumors by age (years) of occurrence

\begin{tabular}{|l|c|c|c|c|c|c|c|}
\hline & \multicolumn{3}{|c|}{ Maxilla } & \multicolumn{3}{c|}{ Mandible } & Mand:Max \\
\hline & class 1 & class 2 & Total & class 1 & class 2 & Total & \\
\hline Ameloblastoma & 3 & 3 & 6 & 4 & 30 & 34 & 5.67 \\
\hline CEOT & 0 & 0 & 0 & 0 & 1 & 1 & NA \\
\hline AOT & 6 & 0 & 6 & 0 & 0 & 0 & NA \\
\hline Ameloblastic fibroma & 0 & 0 & 0 & 0 & 1 & 1 & NA \\
\hline odontoma & 6 & 0 & 6 & 4 & 2 & 6 & 1 \\
\hline
\end{tabular}

CEOT: Calcifying epithelial odontogenic tumor.

AOT: Adenomatoid odontogenic tumor.

NA: Not appliacble

Table 3. Distribution of odontogenic tumors by site of occurrence

mors were most commonly encountered in the posterior zone of the jaws. But adenomatoid odontogenic tumors defied this general finding and were more common in the anterior zone of the jaws.

\section{Discussion}

Available literature on the relative frequency of odontogenic tumors is mostly among Americans, Europeans, and Africans. Very few studies are reported among Asians, especially from the Indian subcontinent.

In this study, all of the odontogenic tumors reported were benign. Among these benign tumors, odontogenic tumors without odontogenic ectomesenchyme were the most commonly encountered $(76.6 \%)$ in this study, which is in agreement with previous reports from India (1), Africa (2), Hong Kong(3), Turkey (4), and China (5). But this finding is in contrast to reports from the U.S.A. (6), Mexico (7), Chile (8), Germany (9), and Canada (10), which reported odontoma as the most commonly encountered odontogenic tumors.

About $99.2 \%$ of OT in the present series were found in patients older than 5 years. Many odontogenic tumors are thought to arise from the tooth germ (1). In most permanent teeth, crown formation completes by the age of 4 or 5 years, which indicates that odontogenic tumors probably develop after crown formation. This strengthens the impression that the majority of odontogenic tumors arise from quiescent remnants of the tooth germ. In the present study, odontogenic tumors were most frequent in the second to fifth decades of life.

Most of the previous studies reported an equal gender distribution of odontogenic tumors but a female preponderance was reported by Regezi et al (6) and Wu and Chan (3); male predominance was reported by Odukoya (2). In the present series, we also found an equal distribution of occurrence between the genders. In general, the odontogenic tumors in this series occured 2.3 times more commonly in the mandible than in the maxilla, which is in agreement with most of the previous studies $(2,5)$.

Ameloblastoma with marked predilection for the mandible was the most frequent tumor (66.67\%) in the present series. This is similar to other studies reported from India (1), Africa (2), Turkey (4), Hong Kong (3), and China (5) , but in contrast to those reported from Canada (10), Chile (8), Germany (9), U.S.A. (6), and Mexico (7) where odontoma is reported as the most common odontogenic tumor. This also strengthens the belief that ameloblastomas are more common in Asians and Africans compared with Caucasians. However, this reported variation may be due to 2 reasons. Over- or under-reporting has a direct influence on this phenomenon. And most odontomas are discovered on routine radiographs and do not produce clinical symptoms (1). This may be responsible for the low incidence of odontomas observed in the Indian population, because most patients in our environment do not seek medical consultation unless there are symptoms suggesting an obvious pathology.

Almost $85 \%$ of ameloblastomas were located in the mandible, with a very high mandible to maxilla ratio (5.67:1). This is similar with the study by Reichart et al (11) who found in an extensive review of all of the cases 
reported in the literature, the ratio to be around 5.4:1. In the present study, ameloblastomas were frequently encountered in the molar-ramus region in the mandible and the molar region in the maxilla.

In the present series, the second most common odontogenic tumor was odontome $(20 \%)$. This is in contrast with the study carried out in canadian population (10), Bhaskar's series (12) and the study carried out in Argentine population (13).

AOT was the third most common tumor. The female predilection of AOT is supported by earlier reports (1, $2,6)$. AOT also showed predilection for the maxilla and anterior regions of the jaws, unlike other odontogenic tumors. Compared with ameloblastomas, AOT occurs at a significantly lower age. Based on the fact of AOT being a well encapsulated tumor that expands centrifugally, i.e. equally in all directions, we hypothesize that AOT may cause expansion of the cortical plates at an early stage compared with ameloblastomas, which spread linearly within the cancellous bone before causing expansion/resorption of the cortical plates. Furthermore, AOTs occur more frequently in the anterior region, which might alert the individual to seek attention at an earlier stage.

Calcifying epithelial odontogenic tumor and ameloblastic fibroma were exclusively diagnosed in females with both having predilection for mandible in this study. They represent $1.67 \%$ of all OT. The frequency of these neoplasms in other series was also lower, confirming the rarity of these tumors.

In conclusion, we observed a marked geographic variation in the relative incidences of various odontogenic tumors. This was particularly notable in ameloblastomas and odontomas, with the incidences observed in the present study being similar to previous studies from India, Africa and Asia and in contrast to those reported from American and European countries.

\section{References}

1. Sriram G, Shetty R P. Odontogenic tumors: a study of 250 cases in an Indian teaching hospital. Oral Surg Oral Med Oral Pathol Oral Radiol Endod. 2008; 105:E14-21.

2. Odukoya O. Odontogenic tumors: analysis of 289 Nigerian cases. J Oral Pathol Med. 1995; 24: 454-7.

3. Wu PC, Chan KW. A survey of tumors of the jawbones in Hong Kong Chinese: 1963-82. Br J Oral Maxillofac Surg.1985; 23: 92-102. 4. Günhan O, Erseven G, Ruacan S, Celasun B, Aydintug Y, Ergun E, et al. Odontogenic tumours. A series of 409 cases. Aust Dent J. 1990;35:518-22.

5. Lu Y, Xuan M, Takata T, Wang C, He Z, Zhou Z, et al. Odontogenic tumors: A demographic study of 759 cases in a Chinese population. Oral Surg Oral Med Oral Pathol Oral Radiol Endod. 1998;86:707-14. 6. Regezi JA, Kerr DA, Courtney RM. Odontogenic tumors: analysis of 706 cases. J Oral Surg. 1978; 36: 771-8.

7. Mosqueda-Taylor A, Ledesma-Montes C, Caballero-Sandoval S, Portilla-Robertson J, Ruíz-Godoy Rivera LM, Meneses-García A. Odontogenic tumors in Mexico: a collaborative retrospective study of 349 cases. Oral Surg Oral Med Oral Pathol Oral Radiol Endod. 1997;84:672-5.

8. Ochsenius G, Ortega A, Godoy L, Peñafiel C, Escobar E. Odontogenic tumors in Chile: a study of 362 cases. J Oral Pathol Med.
2002;31:415-20.

9. Larsson A, Almeren H. Ameloblastoma of the jaws. An analysis of a consecutive series of all cases reported to the Swedish Cancer Registry during 1958-1971. Acta Pathol Microbiol Scand 1978, 86:337-49. 10. Daley TD, Wysocki GP, Pringle GA. Relative incidence of odontogenic tumors and oral and jaw cysts in a Canadian population. Oral Surg Oral Med Oral Pathol. 1994;77:276-80.

11. Reichart PA, Philipsen HP, Sonner S. Ameloblastoma: biological profile of 3677 cases. Eur J Cancer B Oral Oncol. 1995;31B:86-99.

12. Bhaskar S N .Oral pathology in the dental office: survey of 20,575 biopsy specimens. J Am Dent Assoc. 1968; 76:761-6.

13. Guerrisi M, Piloni MJ, Keszler A. Odontogenic tumors in children and adolescents. A 15-year retrospective study in Argentina. Med Oral Patol Oral Cir Bucal. 2007;12:E180-5. 